\title{
A SUBOPTIMAL DETECTION SCHEME FOR MIMO SYSTEMS WITH NON-BINARY CONSTELLATIONS
}

\author{
M. Magarini, A. Spalvieri \\ Dipartimento di Elettronica e Informazione, Politecnico di Milano, \\ Piazza Leonardo da Vinci, 32, I-20133 Milano (Italy), \\ \{magarini,spalvier\}@elet.polimi.it
}

\begin{abstract}
For multiple-input multiple-output (MIMO) spatial multiplexing systems the complexity of the maximum likelihood detector (MLD) can be prohibitively extensive when the number of transmitting antennas and constellation points is high. To simplify the MLD many linear and nonlinear techniques have been proposed. In this paper the principle of reduced state sequence detection, based on mapping by set partitioning, is applied to perform detection in MIMO systems. Simulation results are presented for the proposed suboptimal detection algorithm.
\end{abstract}

Keywords - Antenna arrays, interference suppression, MIMO systems, detection, multilevel modulation.

\section{INTRODUCTION}

The ever increasing demand for high capacity in wireless communications has contributed to consider the introduction of architectures based on multiple antenna elements both for the transmitter and for the receiver [1]. In such schemes, the increase of capacity is achieved by demultiplexing the data sequence in $N$ substreams that are then transmitted, over the multiple input multiple output (MIMO) channel, through $N$ antennas. At the receiver, an estimate of the transmitted data symbols is produced by processing the signals received from $M \geq N$ antennas. The performance of these schemes, in terms of error probability, is strongly dependent on the technique that is implemented in the receiver to detect the $N$ substreams. The optimum detector, which is the maximum likelihood detector (MLD), has a complexity proportional to $q^{N}$, where $q$ denotes the number of constellation points. Due to this exponential dependence the complexity of the MLD can be prohibitively extensive when the number of transmitting antennas and constellation points is high.

The V-BLAST architecture, proposed in [2], is a practical nonlinear detection technique that allows the detection of the $N$ substreams while keeping the complexity low. In such a scheme symbols are detected sequentially according to the well known nulling and successive interference cancellation process. In [3] it is demonstrated that V-BLAST operations are equivalent to those of a decision-feedback equalizer (DFE) that operates at dif- ferent stages in the spatial domain. Hence, the overall performance may be limited by error propagation that takes place in the first stages of the spatial DFE. Despite its detection simplicity, the main drawback of V-BLAST is that the diversity order in the early stages is lower than in the next ones [4]. This contributes to enhance the performance gap between V-BLAST and MLD (in the latter the diversity order is equal to $M$ ).

The performance loss can be mitigated if an appropriate detection ordering is introduced. In [2] and [5] the post-detection signal to interference and noise ratio (SINR) is taken as the ordering criterion. However, this solution may not be sufficient to compensate for low diversity of first stages. Other strategies have been devised to improve the performance of first stages. In [4], for example, the MLD is used to increase the order diversity of first stages and only when the detected symbols are reliable enough the detection is done with the V-BLAST algorithm.

In this paper, we propose a detection technique that is based on the principle of mapping by set partitioning [6]. In our approach the detection problem is decomposed into a series of individual detections for the subsets of the constellation associated to each transmitted substream. Simulation results show that our proposal greatly outperforms the V-BLAST at the cost of a slightly increase in the detector complexity.

The paper is organized as follows. The model of the system we focus on is given in section II. Section III describes V-BLAST detection system with related background. In section IV the proposed detection algorithm is described. Experimental results are then shown in section $\mathrm{V}$ and conclusions are given in section VI.

\section{System Model}

The block diagram of the system under consideration is shown in Fig. 1. From each antenna we consider the transmission of symbols that are drawn from a QAM constellation. The average power radiated from each antenna is fixed to $1 / N$. Thus, the total average radiated power is fixed to 1 and it turns out to be independent of the total number of transmitting antennas. Let $\tilde{\mathbf{a}}=\left[\tilde{a}_{1}, \tilde{a}_{2}, \ldots, \tilde{a}_{N}\right]^{T}$ denote the vector of transmitted symbols, each chosen from a set $\mathcal{A}\left((\cdot)^{T}\right.$ denotes trans- 


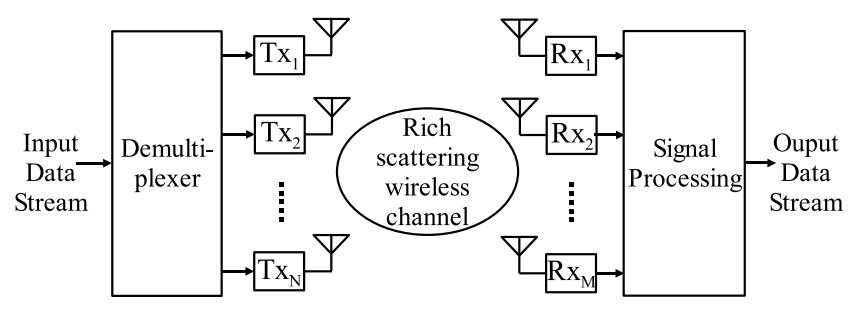

Fig. 1. Block diagram of the V-BLAST system.

position). The vector $\tilde{\mathbf{a}}$ is considered as a point in the $\mathcal{A}^{N}$ space. The corresponding received $M$-dimensional vector is:

$$
\mathbf{r}=\mathbf{H a}+\mathbf{w}
$$

where $\mathbf{w}$ is a vector of i.i.d. complex Gaussian random variables (RV's) with zero mean and variance $\sigma_{w}^{2}$ and $\mathbf{H}$ is the $M \times N$ channel matrix whose elements are i.i.d. RV's having uniform distributed phase and Rayleighdistributed magnitude with average power equal to 1 . $\mathbf{H}$ is independent of both $\tilde{\mathbf{a}}$ and $\mathbf{w}$ and it is assumed perfectly known to the receiver.

\section{BACKGROUND}

In the original implementation of V-BLAST the received vector elements are linearly weighted to null the interference from the yet undetected symbols [2]. This approach leads to the so-called zero-forcing (ZF) VBLAST. It is worth noting that ZF V-BLAST disregards the effects of noise and removes only the interference components. A better performance is obtained if one minimizes the effects of total disturbance, that is, interference plus noise. This approach leads to minimummean square error (MMSE) V-BLAST [5].

In MMSE V-BLAST interference suppression is performed by linearly weighting the received vector in order to minimize the variance of the error between the transmitted vector ã and the receiver's estimate $\hat{\mathbf{a}}$. The error vector is defined as

$$
\mathbf{e}=\tilde{\mathbf{a}}-\hat{\mathbf{a}}=\tilde{\mathbf{a}}-\mathbf{G r},
$$

where $\mathbf{G}$ is the $M \times N$ linear weight matrix. By minimizing the mean square error defined as

$$
\mathrm{MSE}=E\left\{\|\mathbf{e}\|^{2}\right\}=E\left\{\|\tilde{\mathbf{a}}-\mathbf{G r}\|^{2}\right\},
$$

one obtains the following MMSE weight matrix [5]

$$
\mathbf{G}_{\mathrm{MMSE}}=\left(\mathbf{H}^{\dagger} \mathbf{H}+\alpha \mathbf{I}_{N}\right)^{-1} \mathbf{H}^{\dagger},
$$

where $\alpha=N \sigma_{w}^{2}, \mathbf{I}_{N}$ is an $N \times N$ identity matrix and ${ }^{\dagger}$ denotes conjugate transposition. The covariance matrix of the error vector in (1) is

$$
\mathbf{P}=E\left\{\mathbf{e}^{\dagger}\right\}=\left(\mathbf{H}^{\dagger} \mathbf{H}+\alpha \mathbf{I}_{N}\right)^{-1} .
$$

It is worth noting that the MMSE linear matrix $\mathbf{G}_{\text {MMSE }}$ given in (2) introduces bias in the decision process [7], [8]. A better performance is obtained by removing the bias. The unbiased MMSE (UMMSE) linear matrix is given by

$$
\mathbf{G}_{\mathrm{UMMSE}}=\kappa \mathbf{G}_{\mathrm{MMSE}}
$$

where $\kappa=1+\alpha$.

The soft decision statistic for the symbol sent from antenna $i$ is given by

$$
y_{i}=\mathbf{G}_{\mathrm{UMMSE}, \mathrm{i}} \mathbf{r},
$$

where $\mathbf{G}_{\mathrm{UmmSE}, \mathrm{i}}$ is the $i$-th row of matrix $\mathbf{G}_{\mathrm{Ummse}}$. The estimate of the symbol sent by antenna $i$ is obtained as

$$
\hat{a}_{i}=Q\left(y_{i}\right)
$$

where $Q(\cdot)$ denotes the quantization operation appropriate to the constellation in use. With pure disturbance suppression, each component is always detected in the presence of others, so ordering does not matter.

In V-BLAST to alleviate the problem of error propagation an ordering has to be introduced to establish which signal has to be detected first. Clearly, in this case, the best symbol estimate would be the one that corresponds to the smallest error variance, that is, the one for which the $i$-th element of the main diagonal of the error covariance matrix $\mathbf{P}$ in (3) is the lowest. This leads to an ordering criterion where the symbol with the highest SINR is detected first [5]. Let $\mathbf{r}_{1}$ denote the received signal vector $\mathbf{r}$ in this level. In the combined ordered interference suppression and cancellation approach of UMMSE V-BLAST, the receiver starts by computing the symbol estimate $\hat{a}_{i}$ by using (4) and (5). For calculation of the next symbol estimate, the interference contribution of the hard estimate $\hat{a}_{i}$ is subtracted from the received signal $\mathbf{r}_{1}$. Thus, treating $\hat{a}_{i}$ as a known quantity, we obtain the following reduced order problem

$$
\mathbf{r}_{2}=\mathbf{r}_{1}-\mathbf{h}_{i} \hat{a}_{i}=\mathbf{H}^{(2)} \tilde{\mathbf{a}}+\mathbf{w},
$$

where $\mathbf{h}_{i}$ denotes the $i$-th column of $\mathbf{H}$ and $\mathbf{H}^{(2)}$ is the deflated version of $\mathbf{H}$ obtained by setting to zero the $M$ entries of the $i$-th column. This modified received signal, denoted by $\mathbf{r}_{2}$, has a lower level of interference and this will increase the probability of correct detection at the successive stage. The UMMSE solution to (6) requires the computation of (2) for the deflated matrix $\mathbf{H}^{(2)}$ and the corresponding error covariance matrix $\mathbf{P}^{(2)}$. This process continues up to level $N$. On the contrary, it is worth noting that with pure linear UMMSE disturbance suppression, the coefficients of $\mathbf{G}_{\mathrm{UMMSE}}$ are only computed once, as $\mathbf{H}$ remains unchanged. This will reduce system performance but some computational cost can be saved. 


\section{The Proposed Detection Algorithm}

By taking inspiration from reduced state sequence detection [6], based on mapping by set partitioning (MBSP), we propose a detection algorithm for MIMO systems with non-binary constellations. The principle behind MBSP is a geometric partitioning of the constellation in subsets of diminishing size, in such a way that minimum Euclidean distance within the subsets increases down the partition chain. The partition is usually binary at each level. Set partitioning allows to assign binary labels to constellation points according to the subset they are in, and therefore in agreement with their Euclidean distance. The least significant bit (LSB) is assigned according to which of the two subsets a point is in on the first level of partition, the next bit on the next level, and so on until the most significant bit (MSB) identifies the point within the bottom-level subset.

In QAM modulation, the real and imaginary part of each symbol belongs to the integer set $\mathbb{Z}$. In our proposal, the binary partition $\mathbb{Z} / 2 \mathbb{Z}$ is considered in each dimension of the QAM constellation for each transmitted substream. Depending on the constellation size other partitions could be considered. Let $q=2^{2 k}$ be the size of the QAM constellation in use, with $k=1,2, \ldots$ A list of $2^{2 N}$ candidate subsets is generated by considering the $2^{2 N}$ combinations of LSB's for the $N$ entries of the transmitted vector. The UMMSE V-BLAST is applied to perform detection in each of these $2^{2 N}$ subsets containing $2^{2(k-1) N}$ constellation points. If a lower computational cost is desired, the detection can be performed by the linear UMMSE. At each stage the detector examines the decision statistic for the symbol sent from antenna $i$ and compares it with the candidate symbols that are drawn from the current subset associated to substream $i$. At the end of this procedure a list of $2^{2 N}$ candidate vectors is generated. A final decision is taken by applying the MLD to this reduced set

$$
\hat{\mathbf{a}}=\arg \min _{\hat{\mathbf{a}}_{r} \in \mathcal{A}_{r}}\left\|\mathbf{r}-\mathbf{H} \hat{\mathbf{a}}_{r}\right\|^{2},
$$

where $\hat{\mathbf{a}}_{r}$ is a vector taken from the reduced set $\mathcal{A}_{r}$ containing the $2^{2 N}$ candidate vectors. It is worth noting that the complexity of the MLD on the reduced set of candidate vectors is fixed and is independent of the size of the constellation in use. We emphasize that other partitions are possible, but we are interested here in maintaining the complexity of the detector low.

\section{Experimental Results}

In this section we present some simulation results showing the performance of the proposed detection algorithm. We assume that the number of receiving antennas is equal to the number of transmitting antennas, that is $M=N$.

The performance of the proposed detection algorithm, termed SP (set partitioning), is compared with that of

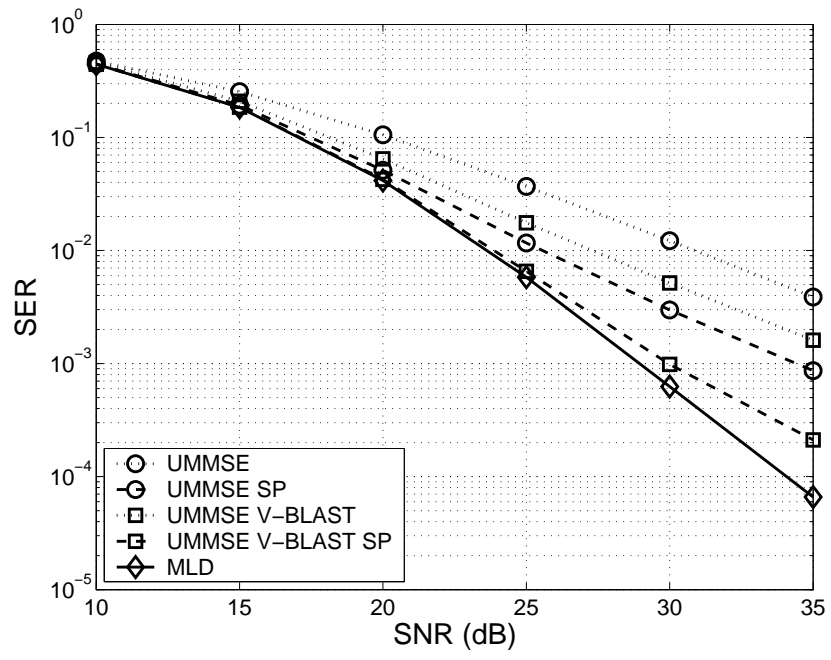

Fig. 2. SER versus SNR for the different detection algorithms in a $2 \times 2$ system using 16-QAM.

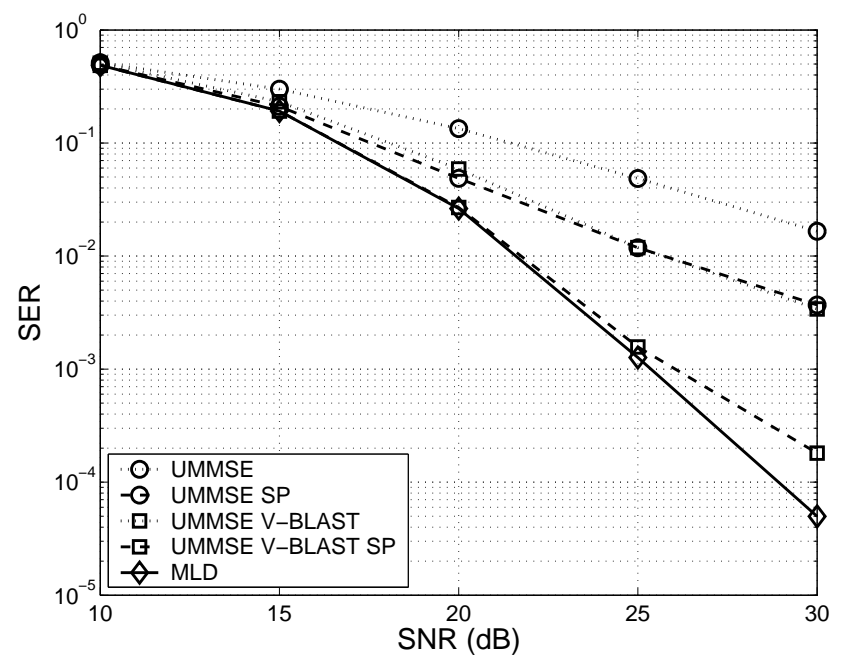

Fig. 3. SER versus SNR for the different detection algorithms in a $3 \times 3$ system using 16 -QAM.

the linear UMMSE and of the UMMSE V-BLAST with ordering. In all the figures, the simulated MLD performance is also reported as a benchmark. Note that, an estimate of the MLD performance could be obtained by using the analytical bound derived in [9]. We consider the application of the SP detection algorithm both with the linear UMMSE (linear UMMSE SP) and with the ordered UMMSE V-BLAST (UMMSE V-BLAST SP) as subset candidate vector detectors. Figures 2 and 3 report the symbol error rate (SER) versus SNR (signal-to-noise ratio, $\left.\mathrm{SNR}=1 / N_{0}\right)$ respectively in a $2 \times 2$ and in a $3 \times 3$ systems using 16 -QAM modulation. The SER is measured by transmitting a sequence of $10^{6}$ QAM data symbols from each antenna. We observe that the performance of the UMMSE V-BLAST SP algorithm is close 


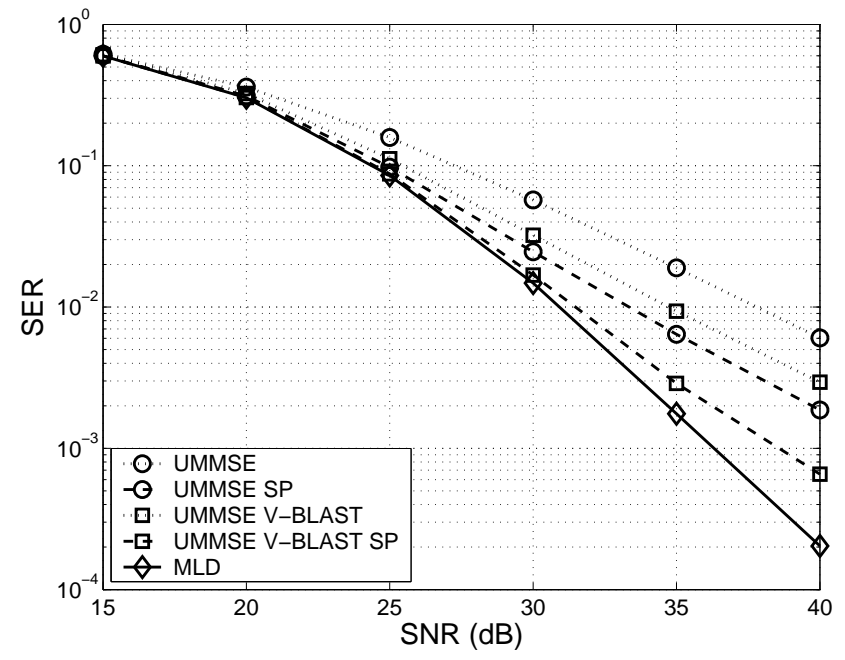

Fig. 4. SER versus SNR for the different detection algorithms in a $2 \times 2$ system using 64 -QAM.

to that of the MLD for a SER greater than $10^{-3}$. Due to a different slope of the curves, for a lower SER the performance of the UMMSE V-BLAST SP detection algorithm deviates from that of the MLD. From the two figures we observe the remarkable performance improvement of UMMSE V-BLAST SP detection technique compared to that of the UMMSE V-BLAST with ordering. It is worth noting that linear UMMSE SP outperforms UMMSE VBLAST with ordering in the $2 \times 2$ system, while they have the same performance for the $3 \times 3$ system. For the same systems, figures 4 and 5 report simulation results when $64-\mathrm{QAM}$ is used. The figures show that performance advantage provided by SP is again consistent. However, in this case the point where the SP UMMSE VBLAST curve deviates from the MLD curve corresponds to a SER of $10^{-2}$.

\section{Conclusions}

This paper presents a detection technique based on the principle of mapping by set partitioning for MIMO systems with non-binary constellations. In the proposed technique the computational complexity is determined by the level of partitioning of the constellation in use. Simulation results are shown to evidentiate the benefits of this suboptimal detection algorithm. We emphasize that for low-to-intermediate SNR the performance of the proposed scheme is comparable to that of the MLD.

In the present paper, the performance is evaluated by computer simulation, while the analysis is left to further study. Another subject of future investigations is the extension of the algorithm to coded systems.

\section{REFERENCES}

[1] B. Vucetic, J. Yuan, Space-time coding, John Wiley \& Sons Ltd, West Sussex, England, 2003.

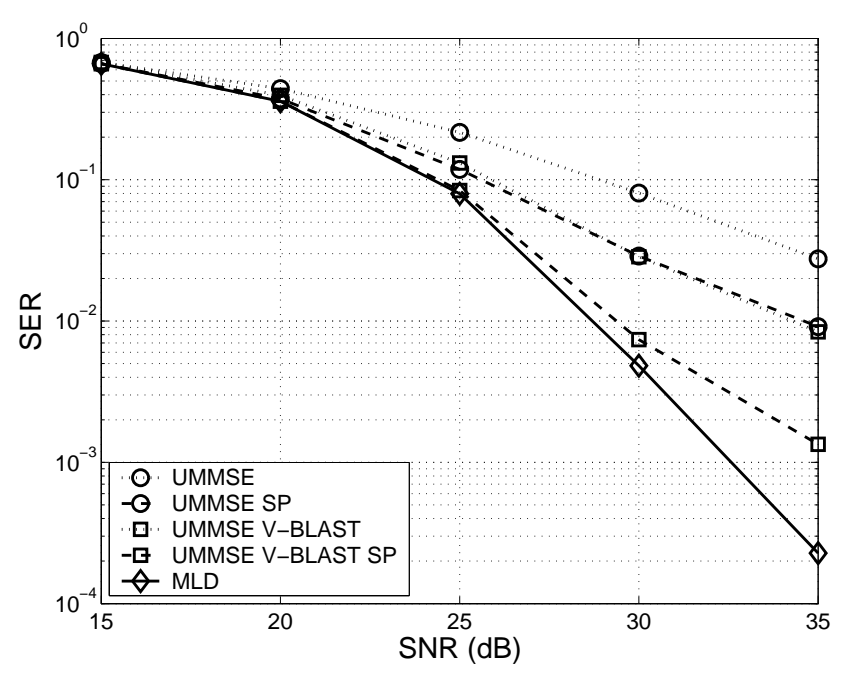

Fig. 5. SER versus SNR for the different detection algorithms in a $3 \times 3$ system using 64 -QAM.

[2] P. W. Wolniansky, G. J. Foschini, G. D. Golden, R. A. Valenzuela, "V-BLAST: an architecture for realizing very high data rates over the rich-scattering wireless channel," in Proc. ISSSE, Pisa, Italy, pp. 295-300, 29 Sept.-2 Oct. 1998

[3] G. Ginis, J. M. Cioffi, "On the relation between VBLAST and the GDFE ," IEEE Commun. Lett., vol. 5, pp. 364-366, Sept. 2001.

[4] W. J. Choi, R. Negi, J. M. Cioffi, "Combined ML and DFE decoding for the V-BLAST system," in Proc. ICC, New Orleans, LA, vol. 3, pp. 1243-1248, 18-22 June 2000.

[5] B. Hassibi, "An efficient square-root algorithm for BLAST," in Proc. ICASSP, Istanbul, Turkey, vol. 2, pp. 737-740, 5-9 June 2000.

[6] M. V. Eyuboglu, S. U. H. Qureshi, "Reduced-state sequence estimation with set partitioning and decision feedback," IEEE Trans. Commun., vol. 36, pp. 13-20, Jan. 1988.

[7] J. M. Cioffi, G. P. Dudevoir, M. V. Eyuboglu, G. D. Forney Jr., "MMSE decision-feedback equalizers and coding-part I: equalization results," IEEE Trans. Commun., vol. 43, pp. 2582-2594, Oct. 1995.

[8] E. Biglieri, G. Taricco, A. Tulino, "Performance of space-time codes for a large number of antennas," IEEE Trans. Inform. Theory, vol. 48 , pp. 1794-1803, July 2002.

[9] X. Zhu, R. D. Murch, "Performance analysis of maximum likelihood detection in a MIMO antenna system," IEEE Trans. Commun., vol. 50, pp. 187-191, Feb. 2002. 